\title{
Levoatrial Cardinal Vein: Occluder Embolization and Complication Management
}

\author{
Ilker Mercan, M.D. ${ }^{1}$, Muhammet Akyuz, M.D. ', Baris Guven, M.D. ${ }^{2}$, Onur Isik, M.D. ${ }^{1}$ \\ Departments of 'Pediatric Heart Surgery and 'Pediatric Cardiology, University of Health Sciences Tepecik Training and Research Hospital, Izmir, Turkey
}

\author{
ARTICLE INFO \\ Received May 8, 2020 \\ Revised July 13, 2020 \\ Accepted August 25, 2020 \\ Corresponding author \\ Ilker Mercan \\ Tel $90-232-433-0810$ \\ Fax 90-232-433-0756 \\ E-mail drilkermercan@hotmail.com \\ ORCID \\ https://orcid.org/0000-0002-0295-2641
}

\begin{abstract}
In rare cases, levoatrial cardinal vein may occur as an isolated condition without additional congenital anomalies. Depending on the direction and flow of the shunt, this pathology may produce symptoms; alternatively, it may be asymptomatic, as in the case presented in this study. In asymptomatic cases, complications, such as paradoxical embolism and brain abscess, can arise later. In the 11-year-old patient whose case is presented here, the levoatrial cardinal vein was asymptomatic and incidentally detected. The percutaneous closure method was applied first. However, by 16 hours after the procedure, the occluder device had embolized to the iliac artery. Emergency surgery was performed; first, the occluder device was removed, and levoatrial cardinal vein ligation was then performed via a mini-thoracotomy. The symptoms, diagnosis, and treatment modalities of isolated levoatrial cardinal vein are discussed in the context of this case described herein.
\end{abstract}

Keywords: Vascular malformations, Embolization, Therapeutic, Minimally invasive surgical procedures

\section{Case report}

The levoatrial cardinal vein (LACV) connects the left superior cardinal vein with the left atrium and drains the left atrium into the venous system in the embryological period. Presence of the levoatrial cardinal vein (PLACV) is a very rare congenital anomaly of the systemic venous return. In the early stages of fetal development, the venous plexus between the embryonic foregut and the cardinal, vitellin, and umbilical veins provides the circulatory connection between the heart and lung and ensures the continuity of blood flow. However, obliteration defects that occur during development in this venous system can cause PLACV as a result of the failure of the LACV lumen to close properly [1].

In the literature, the most common anomalies accompanying PLACV have been reported to be hypoplastic left heart syndrome, cor triatriatum, double-outlet right ventricle, tetralogy of Fallot, ventricular septal defect, and atrioventricular septal defect $[1,2]$. Although LACV, persistent left superior vena cava, and vertical vein may seem like identical pathologies from their definitions, the defini- tion of LACV seems to be particularly compatible with the literature regarding a venous connection between the left atrium and innominate vein, as was present in our case [3].

In this case, percutaneous closure was applied to treat isolated PLACV, which was detected incidentally in a patient who had no symptoms and no additional cardiac anomaly. However, the occluder exited the vein and progressed to the iliac artery after the procedure, causing the patient to develop lower extremity ischemia. He underwent embolectomy and simultaneous left posterolateral thoracotomy, and the LACV was ligated.

An 11-year-old male adolescent presented to the pediatric cardiology outpatient clinic to obtain a report on his health before engaging in sporting activity. No cardiac pathological findings were detected during the physical examination of the patient. On transthoracic echocardiography (TTE), a pattern of flow towards the left atrium was observed in an area that was not appropriate for the pulmonary vein. The pulmonary venous return and coronary sinus anatomy appeared normal. A 3-dimensional computed tomography (CT) scan was performed (Fig. 1). Catheter angiography was then planned for the patient. During an- 
giography, when a radiopaque contrast agent was administered to the innominate vein, it was observed that the solution filled the LACV first and then the left atrium. Oxygen saturation and blood gases were measured during catheterization. The saturation level was $98 \%$ in the pulmonary vein and $94 \%$ in the left atrium. The LACV diameter was measured as $5.8 \mathrm{~mm}$. Transcatheter closure was performed with an Amplatzer vascular plug (AVP4 $8 \mathrm{~mm}$; St. Jude Medical, St. Paul, MN, USA) (Fig. 2). Angiography confirmed the absence of a shunt, and the procedure was successfully completed. However, at the 16th hour after the
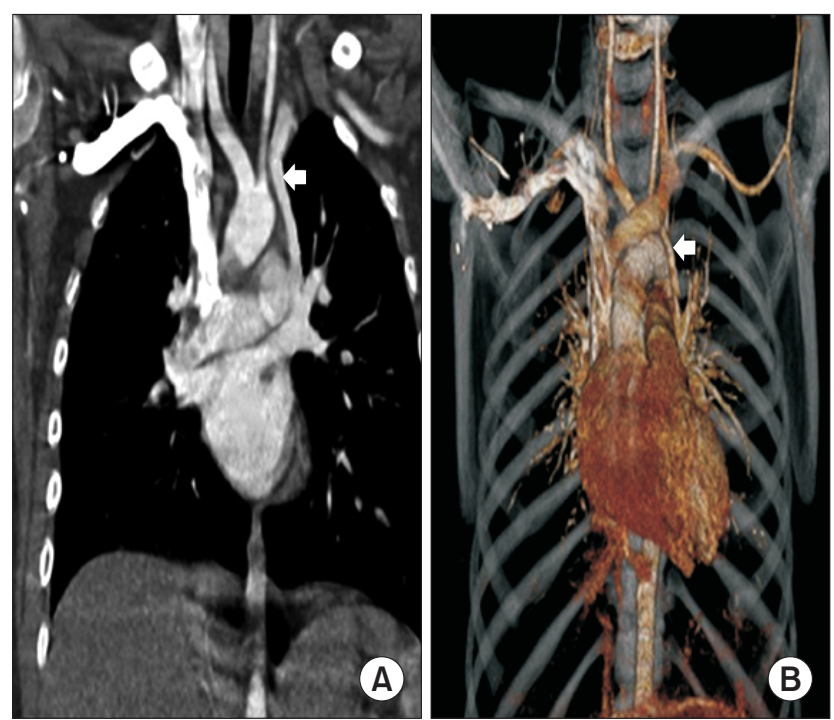

Fig. 1. (A) Computed tomography coronal section view of the levoatrial cardinal vein (arrow). (B) Three-dimensional computed tomography reconstruction view of the levoatrial cardinal vein (arrow). procedure, numbness, coldness, and tingling developed in the patient's right leg. When the patient underwent Doppler ultrasound, monophasic and weak flow was detected in the right main femoral artery. CT angiography was performed, and it was observed that the occluder device had embolized to the right main iliac artery (Fig. 3). The patient was scheduled for emergency surgery. Embolectomy was performed on the right femoral and iliac arteries, and the occluder device was removed. Then, the patient underwent a left posterolateral mini-thoracotomy in the right lateral decubitus position. The LACV was found to extend between the innominate vein and the left atrium. LACV

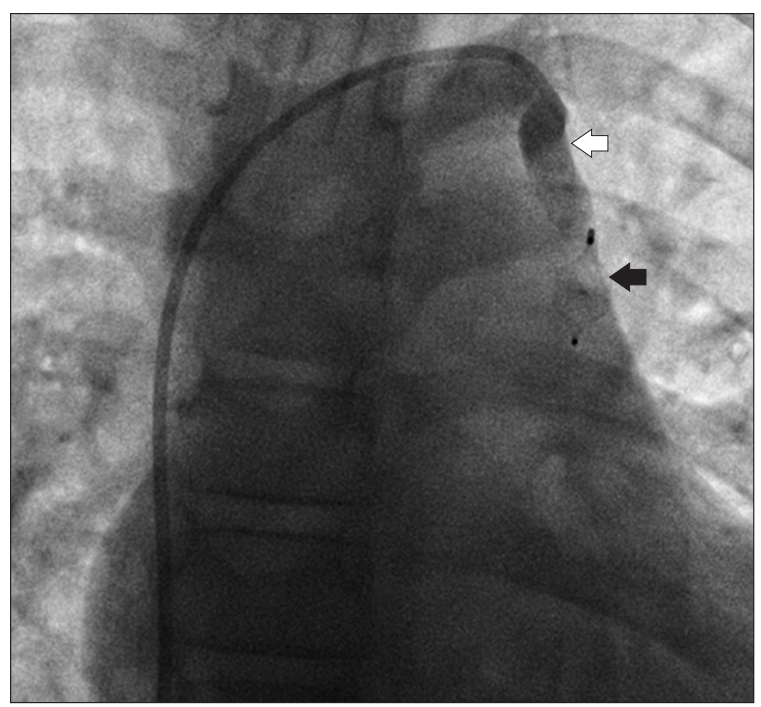

Fig. 2. Catheter angiography view. Contrast transition was not observed after closing the levoatrial cardinal vein with the occluder device (white arrow). Amplatzer plug device (black arrow).

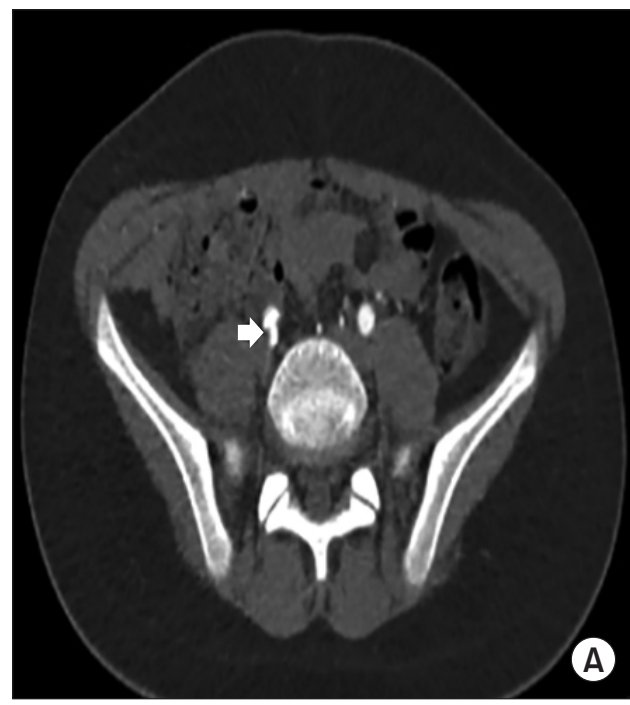

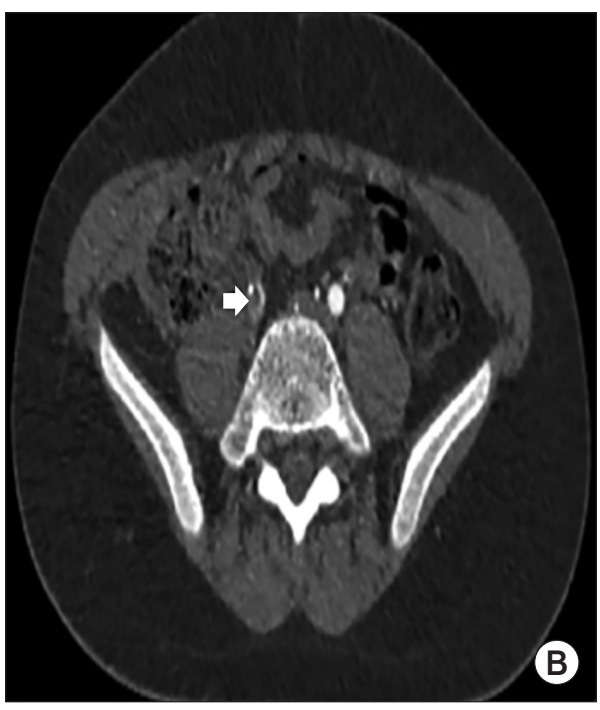

B
Fig. 3. (A) Computed tomography angiography image of the occluder device embolized to the right main iliac artery (white arrow). (B) Contrast filling is not visible in the right main iliac artery (white arrow). 
double ligation and division were applied. The patient was extubated at postoperative hour 3 , and he remained in the intensive care unit 1 day after surgery. The patient exhibited no atriovenous shunt on TTE and was discharged on the third postoperative day after displaying adequate recovery. The patient's consent was obtained for this study.

The patient provided written informed consent for the publication of the clinical details and images associated with his case.

\section{Discussion}

LACV causes shunting between the pulmonary venous circulation and the systemic venous circulation, usually between the left atrium and the innominate vein. PLACV most commonly exists secondary to obstructions on the left side of the heart. In the presence of a congenital malformation (such as severe mitral stenosis) that prevents decompression of the left atrium, the LACV acts as a bridge between the pulmonary and systemic circulation via the innominate vein and provides decompression of the left atrium [3]. Our patient had no congenital anomaly preventing drainage of the left atrium, and the direction of the shunt was from the innominate vein to the left atrium. In most cases presented in the literature, the shunt direction was from the left atrium to the innominate vein. Fujiwara et al. [4] published a report of a PLACV case involving tetralogy of Fallot, and in that case, the left atrial drainage was not impaired. Kaneda et al. [5] also presented an isolated PLACV case. In both cases, the shunt direction was towards the left atrium, and PLACV ligation was preferred as the surgical technique. We employed the double ligation technique used in those rare cases in the literature. We performed mini-thoracotomy due to the absence of accompanying additional cardiac pathology. As such, the patient's recovery process was completed quickly, ensuring that he could return to his daily life rapidly.

Although TTE and catheterization have an important place in the diagnosis of the anomaly described herein, the importance of CT is indisputable. In the literature, the definitive diagnosis of venous return anomalies, which cannot be distinguished by TTE and catheterization, has been clarified with CT scanning $[5,6]$. In the case presented here, we confirmed the diagnosis using 3-dimensional CT after TTE.

PLACV patients may present with symptoms (such as dyspnea, tachypnea, and pulmonary congestion due to additional congenital anomalies) and signs (such as systolic ejection murmur and prominence of the pulmonary vascu- lature), depending on the size and direction of the shunt. In our case, PLACV was detected incidentally and was asymptomatic. In asymptomatic PLACV cases, our opinion is that the shunt should be closed, because the literature reveals that these patients may develop paradoxical embolism and recurrent brain abscesses throughout their lifetimes. This tendency to develop recurrent brain abscesses can be explained by a decrease in the phagocytic and bactericidal contribution of alveolar macrophages secondary to the partial decrease in the participation of systemic venous blood, which is directed to the left atrium with its flow shunted to the pulmonary circulation [7]. Another reason is paradoxical embolism impacting brain tissue [8].

In the literature, transcatheter occlusion has been successfully applied in cases of isolated partial pulmonary venous connection anomaly and PLACV. Cases suitable for percutaneous closure are those that involve patients without additional cardiac anomalies and who no longer require this natural shunt during follow-up after palliative operations, such as the Fontan procedure $[9,10]$. However, we encountered embolization of the occluder device to the iliac artery as a complication. In accordance with the direction of the shunt, the occluder first entered the left atrium and then embolized to the iliac artery. In our patient, the occluder device was placed near the roof of the left atrium by the interventional team. We believe that placing the occluder device close to the innominate vein may prevent the development of complications such as embolization. In addition, avoiding activities that may increase the systemic venous pressure both during and after the procedure may prevent this complication. Considering that surgical correction of this malformation with minimal incisions is quite safe and relatively non-invasive, we believe that this surgical option can be performed safely by experienced practitioners if percutaneous closure fails. The importance of adequate surgical support at centers where percutaneous closure will be performed is also indisputable.

In conclusion, PLACV without any additional left ventricular anomaly is rarely encountered. The symptoms usually seen in cases of PLACV may be associated with other accompanying cardiac malformations or may vary depending on the direction and flow of the shunt. In the present case, the shunt was directed towards the left atrium, since no additional obstructive pathology that would increase the left atrial pressure was present. The convenience of percutaneous closure to the patient is indisputable, but approaches such as surgical ligation through mini-thoracotomy are also very safe and are relatively non-invasive. 


\section{Conflict of interest}

No potential conflict of interest relevant to this article was reported.

\section{ORCID}

Ilker Mercan: https://orcid.org/0000-0002-0295-2641

Muhammet Akyuz: https://orcid.org/0000-0002-5555-564X

Baris Guven: https://orcid.org/0000-0002-4520-5574

Onur Isik: https://orcid.org/0000-0002-6826-8084

\section{References}

1. Hellmund A, Berg C, Herberg U, Geipel A, Kempe A, Gembruch U. Levoatrial cardinal vein in a series of five prenatal cases with hypoplastic left heart syndrome and intact atrial septum. Ultraschall Med 2017;38:206-11.

2. Beckman CB, Moller JH, Edwards JE. Alternate pathways to pulmonary venous flow in left-sided obstructive anomalies. Circulation 1975;52:509-16

3. Cullen EL, Breen JF, Rihal CS, Simari RD, Ammash NM. Levoatrio- cardinal vein with partial anomalous venous return and a bidirectional shunt. Circulation 2012;126:e174-7.

4. Fujiwara K, Naito Y, Komai H, et al. Tetralogy of Fallot with levoatrial cardinal vein. Pediatr Cardiol 1999;20:136-8.

5. Kaneda T, Onoe M, Matsuda M, Moriwaki S, Mori N. Patent levoatrial cardinal vein without left heart hypoplasia. Ann Thorac Surg 2006; 81:740-2

6. Pinto CA, Ho SY, Redington A, Shinebourne EA, Anderson RH. Morphological features of the levoatriocardinal (or pulmonary-to-systemic collateral) vein. Pediatr Pathol 1993;13:751-61.

7. Hoidal JR, Schmeling D, Peterson PK. Phagocytosis, bacterial killing, and metabolism by purified human lung phagocytes. J Infect Dis 1981;144:61-71.

8. Gangadhara MB, Magee AG. Transcatheter occlusion of partial anomalous pulmonary venous connection with dual drainage to left atrium. Ann Pediatr Cardiol 2019;12:144-6.

9. Alcibar J, Gomez S, Vitoria Y, et al. Occlusion of the levoatrial cardinal vein with Gianturco coils after Fontan operation. Rev Esp Cardiol 1999;52:733-6.

10. Ciliberti P, Taylor AM, Yates R, Giardini A. Occlusion of persistent levoatrial cardinal vein without left heart hypoplasia utilizing an Amplatzer device. Eur Heart J Cardiovasc Imaging 2013;14:857. 